\title{
Dynamical behaviors of structural, constrained and free water in calcium- and magnesium-silicate-hydrate gels
}

\author{
Peisi Le ${ }^{\mathrm{a}}$, Emiliano Fratini ${ }^{\mathrm{b}}, \mathrm{Kanae} \mathrm{Ito}^{\mathrm{a}}$ Zhe Wang $^{\mathrm{a}}$, Eugene Mamontov ${ }^{\mathrm{c}}$, Piero Baglioni ${ }^{\mathrm{b}}$, \\ Sow-Hsin Chen ${ }^{\mathrm{a}, *}$ \\ ${ }^{a}$ Department of Nuclear Science and Engineering, Massachusetts Institute of Technology, Cambridge, Massachusetts 02139, USA \\ ${ }^{b}$ Department of Chemistry "Ugo Schiff”' and CSGI, University of Florence, Sesto Fiorentino, Florence I-50019, Italy \\ 'Spallation Neutron Source, Oak Ridge National Laboratory, Oak Ridge, Tennessee 37831, USA
}

\begin{abstract}
Hypothesis

The mechanical properties of cement pastes depend strongly on their porosities. In a saturated paste, the porosity links to the free water volume after hydration. Structural water, constrained water, and free water have different dynamical behavior. Hence, it should be possible to extract information on pore system by exploiting the water dynamics.
\end{abstract}

\section{Experiments}

We investigated the slow dynamics of hydration water confined in calcium- and magnesium-silicate-hydrate (C-S-H and M-S-H) gels using high-resolution quasi-elastic neutron scattering (QENS) technique. C-S-H and M-S-H are the chemical binders present in calcium rich and magnesium rich cements. We measured three M-S-H samples: pure M-S-H, M-S-H with aluminium-silicate nanotubes (ASN), and M-S-H with carboxyl group functionalized ASN (ASN-COOH). A C-S-H sample at the same hydration level (i.e. 0.3) is also studied for comparison.

\section{Findings}

Structural water in the gels contributes to the elastic component of the QENS spectrum, while constrained water and free water contribute the quasi-elastic component. The quantitative analysis suggests that the three components vary for different samples and indicate the variance in the system porosity, which controls the mechanical properties of cement pastes.

Keywords

Water dynamics; Porous material; Quasi-elastic neutron scattering; Calcium-silicate-hydrate; Magnesium-silicate-hydrate.

\section{Introduction}

Concrete, one of the most widely used construction material, is a complex inorganic colloidal system [1]. Portland cement (PC), the most common binder in concrete, is produced from limestone (i.e. calcium carbonate, $\mathrm{CaCO}_{3}$ ) by means of a decarbonation reaction at high temperature that gives $\mathrm{CaO}$. This oxide further reacts with silica source to give alite $\left(\mathrm{Ca}_{3} \mathrm{SiO}_{5}\right)$ and belite $\left(\mathrm{Ca}_{2} \mathrm{SiO}_{4}\right)$ : the calcium silicates binders at the origin of the hydraulic performance of PC. From a stoichiometric point of view, producing one mole of $\mathrm{CaO}$ results in one mole of $\mathrm{CO}_{2}$ at the end of the decarbonation process. Additional $\mathrm{CO}_{2}$ is produced by carbon-rich fuels, such as

\footnotetext{
* Corresponding author. Tel.: +1-617-253-3810; fax: +1-617-258-8863.

E-mail addresses: lepeisi@mit.edu (P. Le), fratini@csgi.unifi.it (E. Fratini), kanae@mit.edu (K. Ito), zwang10@mit.edu (Z. Wang), mamontove@ornl.gov (E. Mamontov), baglioni@csgi.unifi.it (P. Baglioni), sowhsin@mit.edu (S.-H. Chen).
} 
coal, needed to operate the kiln. As a result, cement manufacturing contributes to about $5 \%$ of annual anthropogenic $\mathrm{CO}_{2}$ emissions [2,3]. Thus the search for low-carbon cement as partial or complete replacement of PC has captivated the attention among the scientific community [2-7]. Magnesium-based cements, derived from magnesium orthosilicate to produce $\mathrm{MgO}$, represent one possible alternative to the reduction of $\mathrm{CO}_{2}$ emission connected to $\mathrm{PC}$ production. However, mechanical properties of $\mathrm{MgO}$-based pastes still need to be improved to meet PC standards [2, $6]$.

The binding capability of $\mathrm{CaO}-$ and $\mathrm{MgO}$-based cement is linked to the new solid phases that precipitate from a supersaturated aqueous solution and create a significant area of solid-solid bonding interfaces in a space that was originally occupied by liquid water [2]. The new solid phases are, in PC pastes, primarily calcium-silicate-hydrate (shortly C-S-H) and calcium hydroxide, and in MgO-based cement pastes, magnesium-silicate-hydrate (M-S-H) and magnesium hydroxide. During the hydration process, these solid products gradually become interconnected in a fractal assembly with a certain porosity, which causes the paste to set and gain strength. As a result, hydrated cement pastes contain three kinds of water: structural water bound into the solid phases, constrained water that is strongly absorbed by the surface of gel pores or confined in very small gel pores and free water in the large pores [8]. The mechanical properties of the final hardened cement paste depend strongly on its porosity which in turn in a saturated sample depends on the free water volume of the final product. Ideally, we want the solid phase to replace almost all the initial space occupied by water with only a minimal level of porosity left; such a cement paste or concrete will not only have a high strength, but will also have a low permeability and high durability.

Because of the importance of water in the cement hydration reaction and its relation with the microstructure and mechanical properties of hardened cement pastes, several techniques have been used in the past to gather information on the water state in a non invasive way: neutron scattering [8-19], differential scanning calorimetry (DSC) [20, 21], near infrared spectroscopy (NIR) [21, 22] and nuclear magnetic resonance (NMR) [23] just to cite the most relevant.

Among them, quasi-elastic neutron scattering (QENS) is uniquely suited to study the complex behavior of water in cement paste. A QENS spectrum of hydrated cement pastes is dominated by the scattering from hydrogen because of its large incoherent scattering cross section compared to other elements present in the paste. Hydrogen atoms that are chemically bound to the solid phase (i.e. immobile) contribute only to the elastic component whereas other hydrogen atoms that are in liquid-like (i.e. mobile) water molecules contribute to the quasi-elastic component of a QENS spectrum. Therefore, water at different physical-chemical states can be differentiated by applying a proper model to analyze QENS spectra. The simplest model used is a sum of a delta function representing the signal arising from the chemically bound hydrogen, and a Lorentzian function representing the free water [17]. This model can be further improved by adding a second Lorentzian function to explicitly take into account the constrained water [8, 19]. Both models were successfully used to monitor the evolution of the cement hydration process close ambient conditions while they are not adequate at supercooled temperatures. Theoretical [24, 25], computer simulation [26, 27] and experimental studies [11, 27-32] suggest that in the supercooled regime, the relaxation of the translational motion of water molecules is highly stretched. Therefore, the stretched exponential decay with $\beta<1$ was previously used by us to 
replace the Lorentzian functions thus representing the translational contribution of the liquid water under confinement $[10,31,32]$. This model can fit the measured QENS spectra of cement paste well and thus the structural and liquid water (constrained water and free water) can be studied in detail [10]. In this study, we show that by performing temperature scan on cement pastes at supercooled regime, it is possible to qualitatively compare the constrained water to liquid water ratio (CLR) among different samples. Such comparison is based on the different dynamical behaviors of the constrained water and free water at supercooled temperatures as extracted by QENS analysis. This method can be used to identify the sample with higher CLR which is also considerable as a rough indicator of the average pore size existing in the gel. Although the approach is reported in the case of cement, the present framework could be of potential interest for other porous materials whose hydration water is essential to maintain their colloidal structure or catalytic activity.

\section{Materials and methods}

\subsection{Materials}

Three different samples are studied in this paper. All samples are prepared at the same hydration level $(\mathrm{w} / \mathrm{c}=0.3)$. The first sample is pure M-S-H gel. The second and third samples are M-S-H with $10 \%$ of different additives as reinforcing agents: aluminium-silicate nanotubes (ASN) and carboxyl group $(\mathrm{COOH})$ functionalized ASN (ASN-COOH), correspondingly. A set of data for the C-S-H sample with the same hydration level from Ref. [12] was re-fitted using the same method shown in the following section for comparison.

\subsection{Methods}

\subsubsection{Quasi-elastic Neutron Scattering (QENS)}

QENS experiments were carried out at the high-resolution backscattering spectrometer BASIS [33] at the Spallation Neutron Source (SNS), Oak Ridge National Laboratory (ORNL). BASIS is capable of measuring over a dynamic range near the elastic peak of about $\pm 100 \mu \mathrm{eV}$ in the standard high-intensity operation regime with an elastic energy resolution of $3.4 \mu \mathrm{eV}$ (full width at half-maximum value averaged over all scattering angles). The dynamics of hydration water in silicate gels from initial temperature of $280 \mathrm{~K}$ down to $200 \mathrm{~K}$, varies from picosecond to nanosecond. A time-of-flight backscattering spectrometer such as BASIS is ideal for studying confined water dynamics in supercooled regime because of its wide dynamic range combined with a high energy resolution. The samples were loaded in aluminium foils, which in turn were loaded in cylindrical aluminium sample holders of $29 \mathrm{~mm}$ inner diameter to intercept the $30 \mathrm{~mm}$ by $30 \mathrm{~mm}$ incident neutron beam. The maximum thickness of the sample did not exceed $0.5 \mathrm{~mm}$, corresponding to the minimum transmission of about $80 \%$, in order to both control multiple scattering effects. The instrument resolution functions were measured individually for each sample at $4 \mathrm{~K}$. This is generally necessary for powder samples measured on BASIS, because of possible inhomogeneous distribution of powders in sample holders, since for BASIS the contributions of the sample dimensions to the overall resolution function are significant [33]. Furthermore, even for a perfectly homogeneous sample, the shape of the resolution function is 
rather complex, with a long-time decay due to the neutron moderator pulse shape. The measured resolution functions were normalized before being used in the analysis. Data were reduced to the dynamic structure factors using software available at ORNL also taking into account the subtraction of the empty cell signal.

\subsubsection{Model for Analysis of QENS Spectra}

The intensity of the M-S-H gel at a given $Q$ can be fitted using the equation:

$$
I_{\mathrm{H}_{2} \mathrm{O}}(E)=\left\{p \delta(E)+(1-p) \mathcal{F}\left[F_{S}(t)\right]\right\} \otimes R(E)+B,
$$

where $\mathcal{F}$ denotes a time Fourier transform, $R(E)$ is the measured energy resolution function, $\otimes$ is the convolution operator, $p$ is the elastic fraction, $B$ is a background constant, and $F_{S}(t)$ is the self-intermediate scattering function (SISF). For supercooled water, the decay of $F_{s}(t)$ has two steps [26]. The first step corresponds to some localized motion on a sub-picosecond time scale. This motion is beyond the dynamic range of the spectrometer. The second step (long time) is highly non-exponential and is usually described by a stretched exponential decay form [26]. To account for these considerations, the experimental SISF is modeled with the well-known Kohlraush-Williams-Watts function:

$$
F_{S}(t) \approx \exp \left[-\left(\frac{t}{\tau}\right)^{\beta}\right],
$$

where $\tau$ is the characteristic relaxation time of the long-time relaxation, and $\beta$ is the stretching exponent. The mean characteristic relaxation time $\langle\tau\rangle$ is then calculated by the equation:

$$
\langle\tau\rangle=\beta^{-1} \Gamma\left(\beta^{-1}\right) \tau .
$$

\section{Results and discussion}

\subsection{The physical picture of gel structure}

Before we can discuss the dynamics of confined water, we need to have a physical picture of the microstructure of the C-S-H and M-S-H gels. A previous study [6] has investigated the multiscale stucture of both C-S-H and M-S-H gels using wide-angle X-ray scattering (WAXS), small-angle X-ray scattering (SAXS) and scanning electron microscopy (SEM). The C-S-H and M-S-H are both composed of colloidal units $[6,34]$. The main difference is inside these colloidal units at nanoscale. The building blocks, or globules, of C-S-H are multilamellar discoidal colloids (i.e. silicate-rich layers alternating to water-rich layers) with an average thickness of about $4 \mathrm{~nm}$ and a diameter of about $20 \mathrm{~nm}$, while in M-S-H they are polydisperse sphere colloids with a average diameter of $3.5 \mathrm{~nm}$ and a distribution ranging from 1 to $10 \mathrm{~nm}$. These globules, in turn, condense to form low density (LD) clusters $[6,35,36]$ and generate Large Gel pores (LGP, 3-10 $\mathrm{nm}$ ) and Small gel pores (SGP, 1-3 nm). This is consistent with the differential scanning calorimetry (DSC) thermograms for M-S-H that show two broad peaks, a first peak around 233 $\mathrm{K}$ and a second peak around $227 \mathrm{~K}$ (Fig. 1). In analogy to the Colloidal Model-II (CM-II) for the C-S-H [11, 35-38], the peaks visible in the cooling part of the thermogram can be assigned to SGP. The contribution of the two peaks is different for different samples indicating variance in the obtained pore system. 
With this picture in mind, we can then discuss the dynamics of three water components in C$\mathrm{S}-\mathrm{H}$ and M-S-H gels distinguished by QENS. The first kind of water is the structural water [8]: the water that chemically reacts with the cement powder and becomes a integral part of the reaction products forming the solid matrix. The second kind of water is called constrained water which consists of water confined into the smallest gel pores and water absorbed on the pore surface [8]: in other words, the water physically bound by the matrix. The third kind of water is the free water that is contained in large gel pores or in the capillary porosity.

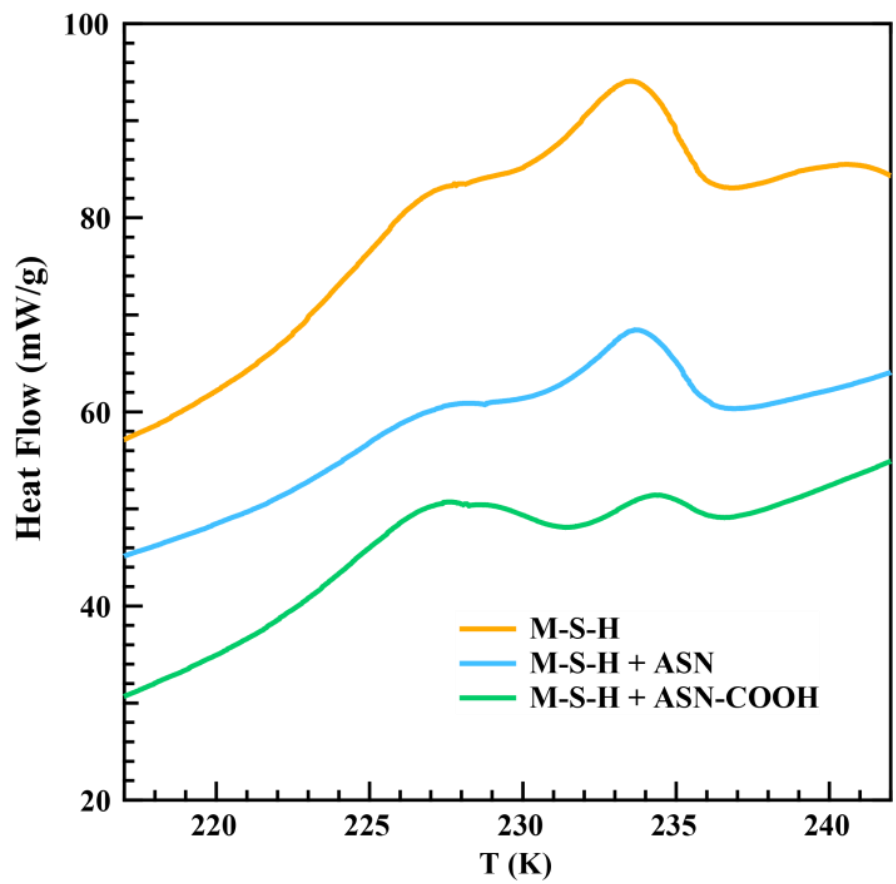

Fig. 1. The DSC thermograms (cooling scan) measured for M-S-H and M-S-H with 2 different additives. In pure M-S-H, it shows two broad peaks, one at $233 \mathrm{~K}$ and one at $227 \mathrm{~K}$. The former is weakened in the case of M-S-H with COOH functionalized aluminium-silicate nanotubes (M-S-H + ASN-COOH).

To separate the different water components in the QENS spectrum, we use the fact that they have different dynamics. When a neutron collides with a chemically bound hydrogen atom, the collision is elastic. There is no energy gained or lost. Thus neutrons will arrive at the detector with zero energy transfer. When a neutron collides with a hydrogen atom in liquid water, the collision is quasi-elastic or inelastic. The neutron energy may change. This occurs because liquid water molecules are mobile: they are constantly translating and rotating. Whether the neutron energy can increase or decrease depends on the relative motion of the hydrogen atom and neutron at the moment of the collision. These neutrons therefore arrive at the detector with a range of energy transfers. If the intensity of scattered neutrons is plotted as a function of their energy transfers, the elastically scattered neutrons generate a sharp peak at zero energy transfer, while the quasi-elastically or inelastically scattered neutrons generate a broad peak that is centered on the zero energy transfer. Thus with an appropriate model for the elastic and the quasi-elastic components, we can separate the structral (chemically bound) water from the liquid (constrained plus free) water in a QENS spectrum. However, to further separate the constrained water from the free water is hard. The commonly used double Lorentzian model for room or 
higher termperatures is not adequate in this study due to the supercooled temperatures. We will first show some results from the elastic scan and the separation of elastic component. Then we will discuss the way to differeniate the constrained water and free water by their different temperature dependent mean relaxation times.

\subsection{Elastic scattering and structural water}

Fig. 2 shows the dynamics of the hydration water in pure M-S-H gel probed using BASIS in the regime of the elastic intensity scan (red circles) which can yield the temperature dependence of the atomic (mainly hydrogen) mean squared displacements (MSDs) (blue squares). To compute the MSDs we used the Q-dependent elastic intensities, $I(Q, T)$ which in the Gaussian approximation yield the MSDs as follows: $\left\langle x^{2}(T)\right\rangle=\left(-3 / Q^{2}\right) \ln \left[I(Q, T) / I\left(Q, T_{0}\right)\right]$, where $I\left(Q, T_{0}\right)$ is the intensity measured at the lowest temperature. A sudden apparent enhancement in the MSDs is observed at a certain temperature threshold. This is a common feature in hydration water when the hydration level is sufficiently high. In the bulk-like state, the dynamic processes involving the "cage-breaking" become observable above certain temperatures [39]. The phenomena indicate that in pure M-S-H gel there is a significant number of free water molecules.

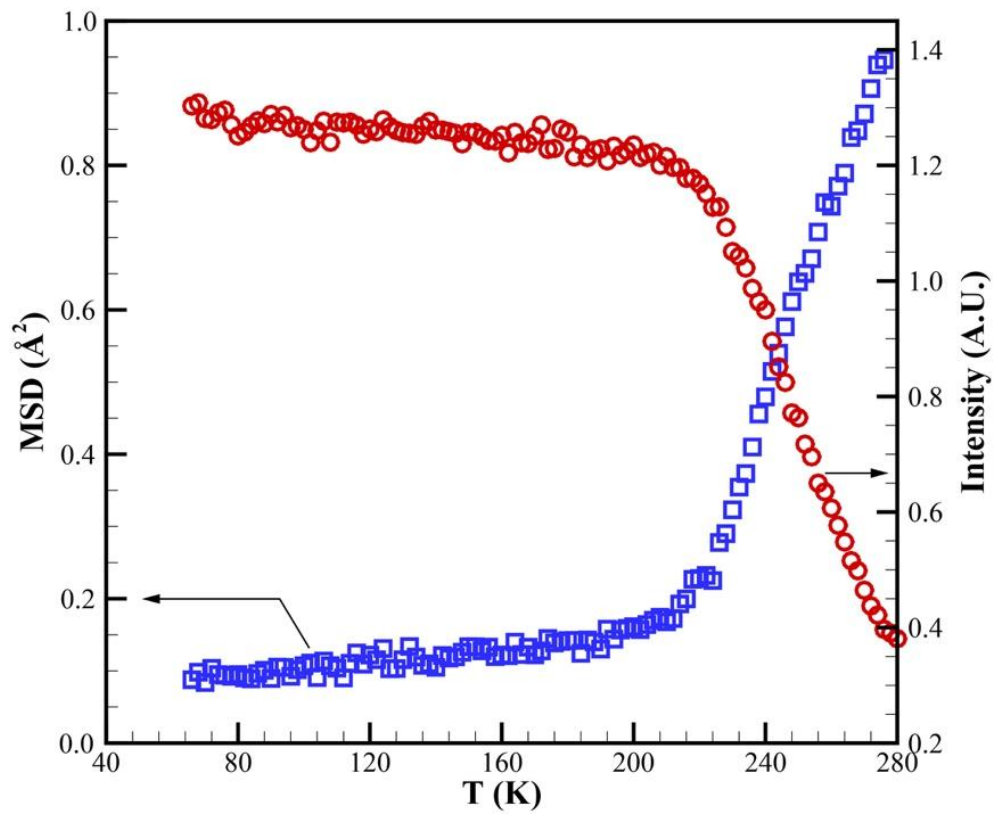

Fig. 2 Red circles represent the variation of the elastic scattering intensity during heating averaged for $0.4 \AA^{-1}<\mathrm{Q}<1.8 \AA^{-1}$ in pure M-S-H gel. Blue squares are mean squared displacements (MSDs) extracted at each temperature.

Fig. 3 shows examples of data analysis using the model described in section 2. Two temperatures are shown, $280 \mathrm{~K}$ and $220 \mathrm{~K}$, at wave vector transfer $Q=0.5$ and $0.7 \AA^{-1}$. The figures are plotted on a semi-log scale to show the good agreement between the model and experimental spectra. The elastic scattering component (green dashed dotted line) originating from the scattering of structural water molecules is separated from the quasi-elastic scattering component (blue dashed line) mainly from scattering of liquid water. The elastic fraction $p$ is equal to the ratio between the structural water and the total water that we can define as the 
structural water index (SWI). SWI is slightly different from the bound water index (BWI) already reported in Ref. [8], that is the ratio of the sum of structural and constrained (chemically and physically bound) water to total water. SWI should not be a function of temperature. Therefore, in the analysis, we fit the $p$ at the highest temperature and keep the value for all the other temperatures for the same sample. The choice to start with the spectra at high temperature to have a reliable estimation of $p$ rely on the fact that the two components become less and less distinguishable as the temperature decreases (see for example Fig. 3 (a) and (c)).
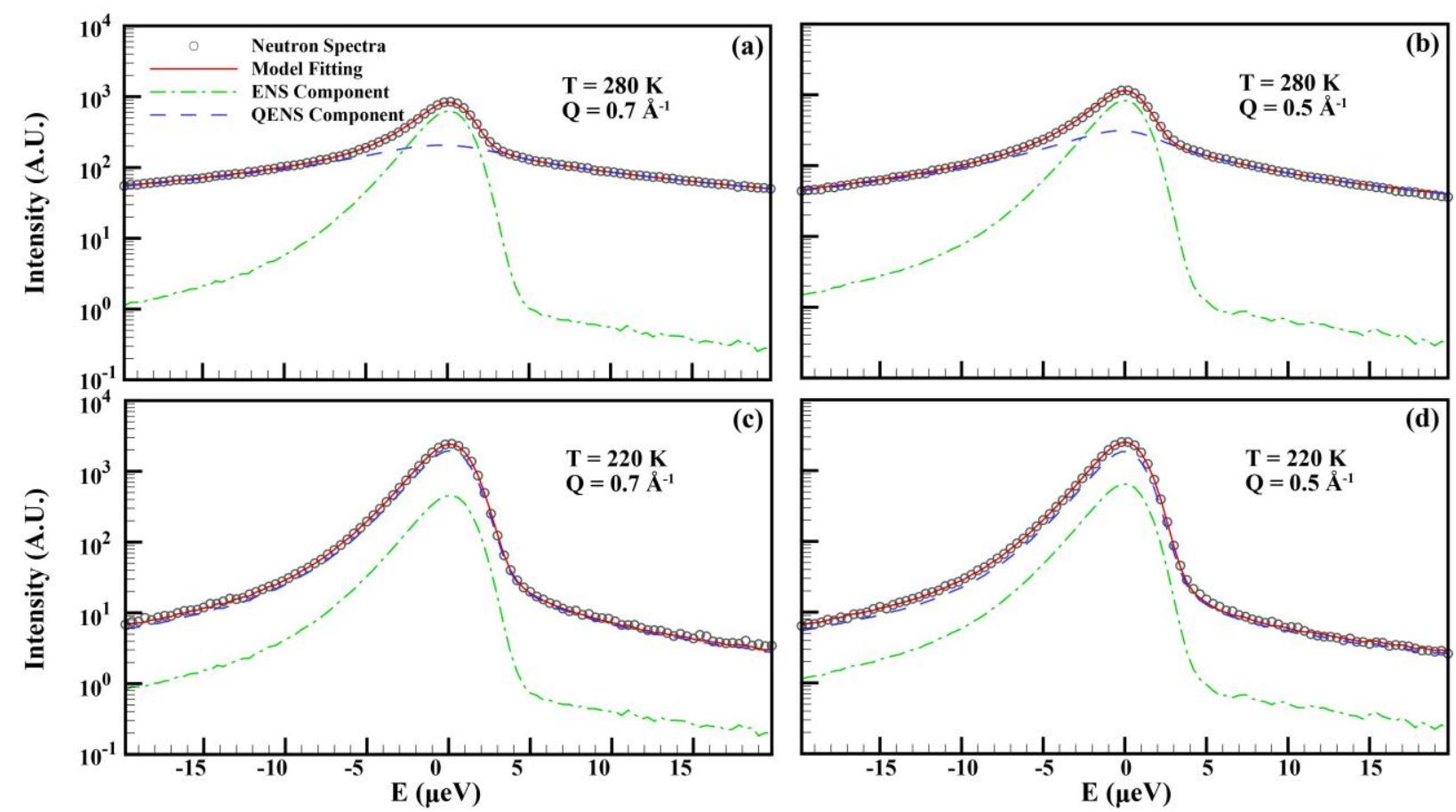

Fig. 3. QENS spectra for pure M-S-H gel at two typical temperatures T $=280 \mathrm{~K}, 220 \mathrm{~K}$ and two wave vectors $Q=0.5$ and $0.7 \AA^{-1}$, respectively. The hollow circles are the measured neutron intensity as a function of the energy transfer $E$. The solid lines are the fitted curves using the model. The dash-dotted green line is the elastic scattering component with asymmetric shape derived from the asymmetric Q-dependent resolution function. The dashed blue line is the quasi-elastic scattering component.

Previous investigations on cement hydration kinetics show that the structural water index exhibits a gradual and continuous increase and mimicking the trend of the heat of hydration as measured by thermal calorimetry [8]. This means that the SWI is proportional to the hydration reaction or the degree of hydration that in turn has a positive correlation to the compressive strength of the cement paste. This is because the strength of cement paste depends primarily on the amount of capillary porosity, and the amount of capillary porosity decreases in proportion to the amount of hydration product that has taken place. A higher SWI indicates more hydration products and less capillary pores thus better compressive strength and lower permeability. The SWIs derived from the elastic fractions for the investigated samples are: $16.7 \%$ for $\mathrm{M}-\mathrm{S}-\mathrm{H}, 21.7 \%$ for M-S-H+ASN and 25.2\% for M-S-H+ASN-COOH. The C-S-H data from Ref. [12] is also refitted using the same model introduced in section 2 for comparison. The C-S-H sample has a SWI of $65.7 \%$, which is about 4 times higher than that of the pure M-S-H sample at the same hydration level of 0.3. Among the three M-S-H samples, the ones with ASN additives, especially 
the one with ASN-COOH, shows higher SWI than pure M-S-H. Thus we conjecture that the ASN additives could improve the compressive strength of magnesium-based cement.

\subsection{Analysis of constrained and free water}

Three parameters are usually important to define a pore system: the total porosity, the pore size distribution and the specific surface area. The SWI is a good indicator of the total porosity since higher SWI corresponds to lower porosity. However, it does not provide information of pore size distribution or the specific surface area. Two cement pastes with the same total porosity can have completely different pore size distribution and specific surface area. The specific surface area that is defined as the area of the interface between solid phases and the pore system per unit amount of material is particularly important in the cement field. It has been shown that the kinetics of the SANS surface area development are very similar to those of the constrained water development measured by QENS [8, 40-42]. This is not surprising since the constrained water is mainly the first and second layer of water physically bound to the surface of the colloids. Therefore, separating the constrained water from the free water in a QENS spectrum can provide us information about the surface area and the pore system. However, this is not as straightforward as the separation of structural water. Previously, the double Lorentzian model has been used for the data analysis of $\mathrm{C}_{3} \mathrm{~S}$ pastes hydrated at $20{ }^{\circ} \mathrm{C}, 30{ }^{\circ} \mathrm{C}$ and $40{ }^{\circ} \mathrm{C}$ [8]. At supercooled temperatures this model may not be adequate as discussed above since in this case the relaxation becomes stretched and non-exponential. In principal, two stretched exponential decays could correctly separate the two contributions. Even if this assumption is theoretically reasonable a single stretched exponential decay already provides a perfect fit for the QENS spectra as seen in Fig. 3 and using two stretched exponentials would result in overfitting thus obtaining unphysical parameters. To circumvent this problem instead of quantitatively separate the two contributions, we introduced a new way to qualitatively identify the sample with the higher constrained water ratio by using an Arrhenius plot. 


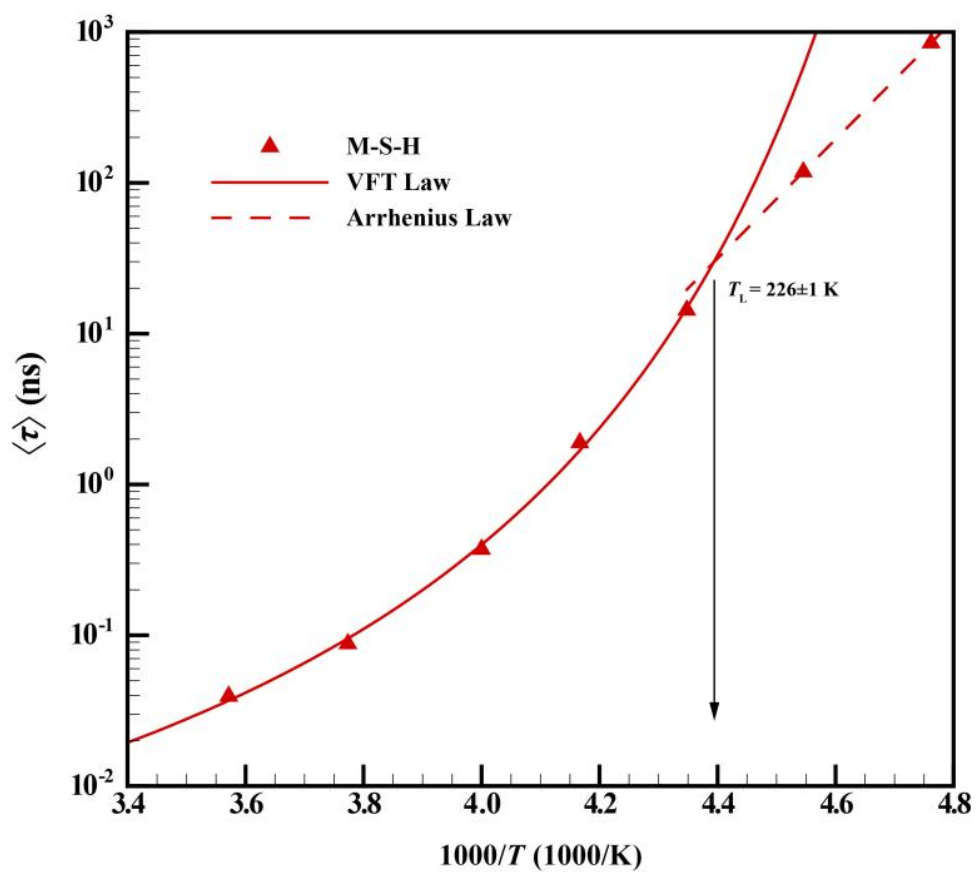

Fig. 4 The Arrhenius plot of the mean characteristic relaxation time $\langle\tau\rangle$ extracted from the QENS spectra at seven temperatures for the pure M-S$\mathrm{H}$ sample with $\mathrm{Q}=0.7 \AA^{-1}$. A super-Arrhenius (non-linear behavior) to Arrhenius (linear behavior) dynamic crossover is observed as the temperature is decreased through $T_{\mathrm{L}}=226 \pm 1 \mathrm{~K}$. The solid line is the fit of the VFT relation. Dashed line is a straight line passing through the only two available points in low temperature region.

Fig. 4 shows the Arrhenius plot of the mean characteristic relaxation time $\langle\tau\rangle$ for the pure M$\mathrm{S}-\mathrm{H}$. At high temperature data exhibits super-Arrhenius behavior, which can be fit by the VogelFulcher-Tammann relation $\langle\tau\rangle=\tau_{0} \exp \left[D T_{0} /\left(T-T_{0}\right)\right]$, while at low temperature data exhibits Arrhenius behavior, which follows the Arrhenius relation $\langle\tau\rangle=\tau_{0} \exp \left(E_{a} / k_{B} T\right)$ [31]. In this experiment, our focus is the high temperature region from $280 \mathrm{~K}$ to $230 \mathrm{~K}$. As shown in Fig. 4, the low temperature region has only two points, which is not enough for a linear fitting. We nevertheless calculated the parameter $E_{a}$ according to the Arrhenius relation substituting these two points. The line passing through the two low temperature points is shown mainly to identify a transition. This transition in $\langle\tau\rangle$ of the hydration water molecule from an Arrhenius (linear) behavior at low-temperature to a super-Arrhenius (non-linear) behavior at high-temperature is called the dynamic crossover. For pure M-S-H at ambient pressure, the dynamic crossover takes place at $T_{\mathrm{L}}=226 \pm 1 \mathrm{~K}$. This is also a common feature in various other hydrated systems $[11,31$, $32,43]$. The non-linear super-Arrhenius behavior happens for hydration water when an adequate fraction of free water molecules enables the "cage-breaking" translational jumps. As the free water fraction increases the super-Arrhenius behavior results stronger. This is because the free water can provide a relatively more complete tetrahedral hydrogen bond network than the constrained water. Therefore, as temperature decreases, the relaxation time of the free water molecules increases more rapidly than that of the constrained water, due to the stronger local order of the former. A similar phenomenon is also observed in studies on hydration dependence of systems such as rutile [39], MCM-41 [44] and lysozyme [31]. Here we define the quantity CLR as the constrained to liquid water ratio (where liquid means the sum of the constrained and free water fractions). Therefore, based on above considerations, we could conclude that smaller 
CLR will result in stronger super-Arrhenius behavior, as a result of the more complete tetrahedral hydrogen bond network present in the free water.

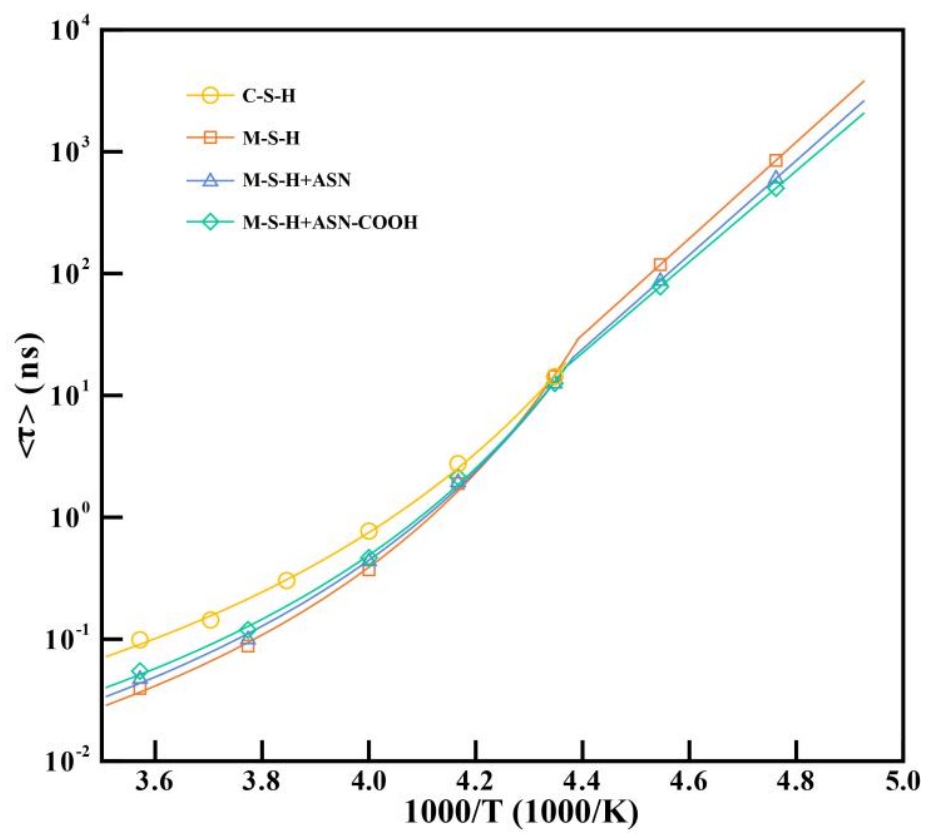

Fig. 5. The figure shows Arrhenius plots of the mean characteristic relaxation time $\langle\tau\rangle$ for M-S-H in this study and C-S-H in Ref. [12] at the same hydration level $(0.3)$ for $\mathrm{Q}=0.7 \AA^{-1}$. The solid lines are the VFT relation at high-temperature and the Arrhenius relation at low-temperature. The variance of VFT fitting curvatures are attributed to the different constrained water to liquid water ratio among samples.

Fig. 5 shows the corresponding Arrhenius plots for the three M-S-H samples and the C-S-H sample at $\mathrm{Q}=0.7 \AA^{-1}$. As discussed above, for the description of the high-temperature region (i.e. $T>220 \mathrm{~K}$ ) we used a VFT relation; conversely the two points in low-temperature region were connected with a straight line applying the Arrhenius relation. The parameters $D, T_{0}$, and $E_{a}$ for the four samples are listed in Table 1 . The parameter $D$ can be used to compare the superArrhenius behavior among different samples. The stronger the super-Arrhenius behavior, the smaller is the value of $D$. All three M-S-H samples exhibit a super-Arrhenius behavior at high temperature (> $226 \mathrm{~K})$. However, the pure M-S-H exhibits the strongest super-Arrhenius behavior among all the samples and has the smallest $D$ of 2.18. This suggests that pure M-S-H has smaller CLR than M-S-H with ASN additives. Another observation is that the C-S-H has the largest $D$ of 2.50, which suggests the studied C-S-H sample has larger CLR than all M-S-H samples.

\begin{tabular}{|c|c|c|c|}
\hline Sample & $D$ & $T_{0}(\mathrm{~K})$ & $E_{a}(\mathrm{kcal} / \mathrm{mol})$ \\
\hline C-S-H & 2.50 & 183 & - \\
\hline M-S-H & 2.18 & 191 & 18.1 \\
\hline $\mathrm{M}-\mathrm{S}-\mathrm{H}+\mathrm{ASN}$ & 2.43 & 187 & 17.8 \\
\hline $\mathrm{M}-\mathrm{S}-\mathrm{H}+\mathrm{ASN}-\mathrm{COOH}$ & 2.43 & 186 & 17.1 \\
\hline
\end{tabular}


From our physical picture of the cement colloidal structure and previous SANS and QENS studies $[8,41]$ the amount of constrained water is approximately proportional to the surface area of the matrix and the amount of liquid water is proportional to the pore volume. Thus the CLR is proportional to the surface-area-to-pore-volume ratio. If we assume that the constrained water has the same average thickness allover the sample, then the CLR can be considered as a rough indicator of the average pore size: i.e. larger CLR correspond to smaller average pore size. Therefore, our study suggests that compared to pure M-S-H, M-S-H with ASN additives may have smaller average pore size which is closer to the C-S-H sample in Ref. [12] at the same hydration level of 0.3 .

Finally, we want to emphasize that although we have discussed the idea mainly based on the hydrated gels in cement, it should also be viable and useful for other porous materials whose hydration water is essential to maintain their colloidal structure or catalytic activity. The basis of the analysis is the common feature of water dynamics found in many other hydrated porous materials such as rutile [39], MCM-41 [44]. In a saturated porous material, the interfacial water attached to the pore surface and the bulk free water located at pore center are different regarding dynamics. Consequently, quantifying the pore solution by its dynamics gives the information of the pore itself. Our study shows that the Arrhenius plot of the mean relaxation time is the right tool to show this difference qualitatively. And a quantitative framework with the same idea is being developed.

\section{Conclusions}

For porous materials, such as cement, characterization of pore is often a better predictor of performance than the characterization of the solid phases [45]. To investigate the pore system of hydrated porous materials, most standard techniques require that removal of pore fluid, but such a drying pretreatment can modify a fragile porous structure $[36,46]$. As one of the non-invasive methods [8-23], QENS is previously used to monitor the state of water and estimate the hydration kinetics of cement [47, 10]. QENS is also used to study the dynamics of water that confined in various porous materials such as MCM-41 [28, 29], rutile (TiO2) [39] and cement [11-13]. From these studies, we know that water located at different pore structure has different physical-chemical states. Hence, it should be possible to extract information on pore systems by exploiting water dynamics.

In this study, the structural water index (SWI), which is closely related to the cement mechanical properties, is extracted from the elastic fraction of the QENS spectrum. Synthetic M$\mathrm{S}-\mathrm{H}$ and $\mathrm{C}-\mathrm{S}-\mathrm{H}$ gels are compared being the principal binder phases for $\mathrm{MgO}$ - and $\mathrm{CaO}-$ based cement. Two M-S-H samples have been prepared with structure reinforcing additives based on aluminium-silicate nanotubes (ASN and ASN-COOH). All the three M-S-H samples have less structural water than C-S-H sample at the same hydration level (i.e. 0.3). M-S-H with ASN and especially ASN-COOH additive, have higher structural water index than pure M-S-H potentially indicating better compressive strength. Different dynamical behaviors between constrained and free water in the Arrhenius plot at supercooled temperatures are used to identify the sample with higher constrained water to liquid water ratio. The sample with lower CLR exhibits a stronger super-Arrhenius behavior. Its characteristic relaxation time results faster at high temperatures 
and slower at low temperatures since at low temperatures the "cage-breaking" translational jumps of free water molecules in a tetrahedral hydrogen bonded network requires longer time [31, 39, 44]. Applying this evidence, we conjectured that M-S-H with ASN additives has higher CLR than pure M-S-H and that C-S-H sample has higher CLR than all the M-S-H samples. The CLR can be seen as a rough indicator of the average pore size assuming constrained water has the same average thickness among all the samples. In this regard, M-S-H with ASN additives could result in smaller average pore size than pure $\mathrm{M}-\mathrm{S}-\mathrm{H}$ and closer to the investigated C-S-H sample. Through SWI and CLR, we introduced a new perspective of comparing the pore systems of hydrated porous materials in a wet state by exploiting water dynamics. This qualitative analysis is the first step of a possible quantitative framework able to provide a way to detail the system porosity without drying the paste, thus approaching the sample in a non-destructive way.

\section{Acknowledgements}

The research at MIT was supported by DOE grant DE-FG02-90ER45429. The neutron scattering experiments at SNS, ORNL were supported by the Scientific User Facilities Division, Office of Basic Energy Sciences, U.S. Department of Energy. E.F. and P.B. acknowledge financial support from Consorzio per lo Sviluppo dei Sistemi a Grande Interfase (CSGI) and MIUR. P. L. thanks Prof. H. Li for providing the C-S-H QENS data and Dr. W.-S. Chiang for her insight on cement structures.

\section{References}

[1] F. Ridi, E. Fratini, P. Baglioni, J. Colloid Interface Sci. 357 (2011) 255-264.

[2] E.M. Gartner, D.E. Macphee, Cem. Concr. Res. 41 (2011) 736.

[3] J.H. Sharp, D.E. Macphee, E.M. Gartner, Adv. Cem. Res. 22 (2010) 195.

[4] M.C.G. Juenger, F. Winnefeld, J.L. Provis, J.H. Ideker, Cem. Concr. Res. 41 (2011) 1232.

[5] M. Liska, A. Al-Tabbaa, Constr. Build. Mater. 22 (2008) 1789.

[6] W.-S. Chiang, G. Ferraro, E. Fratini, F. Ridi, Y.-Q. Yeh, U.-S. Jeng, S.-H. Chen, P. Baglioni, J. Mater. Chem. A 2 (2014) 12991.

[7] H.S. Müller, R. Breiner, J.S. Moffatt, M. Haist, Procedia Eng. 95 (2014) 290.

[8] J.J. Thomas, S. A. FitzGerald, D. A. Neumann, R. A. Livingston, J. Am. Ceram. Soc. 84 (2001) 1811.

[9] D.H.C. Harris, C.G. Windsor, C.D. Lawrence, Mag. Concr. Res. 26 (1974) 65.

[10] E. Fratini, S.-H. Chen, P. Baglioni, M.-C. Bellissent-Funel, J. Phys. Chem. B 106 (2002) 158.

[11] Y. Zhang, M. Lagi, F. Ridi, E. Fratini, P. Baglioni, E. Mamontov, S.H. Chen, J. Phys. Condens. Matter 20 (2008) 502101.

[12] H. Li, E. Fratini, W.-S. Chiang, P. Baglioni, E. Mamontov, S.-H. Chen, Phys. Rev. E 86 (2012) 061505.

[13] H. Li, W.-S. Chiang, E. Fratini, F. Ridi, F. Bausi, P. Baglioni, M. Tyagi, S.-H. Chen, J. Phys. Condens. Matter 24 (2012) 064108.

[14] E. Fratini, A. Faraone, F. Ridi, S.-H. Chen, P. Baglioni, J. Phys. Chem. C 117 (2013) 7358.

[15] H. Li, L.-L. Zhang, Z. Yi, E. Fratini, P. Baglioni, S.-H. Chen, J. Coll. Interf. Sci. 452 (2015) 2.

[16] A.J. Allen, J.C. McLaughlin, D.A. Neumann, R.A. Livingston, J. Mater. Res. 19 (2004) 3242.

[17] S.A. FitzGerald, D.A. Neumann, J.J. Rush, D.P. Bentz, R.A. Livingston, Chem. Mater. 10 (1998) 397.

[18] S.A. FitzGerald, D.A. Neumann, J.J. Rush, R.J. Kirkpatrick, X. Cong, R.A. Livingston, J. Mater. Res. 14 (1999) 1160.

[19] S.A. FitzGerald, J.J. Thomas, D.A. Neumann, R. Livingston, Cem. Concr. Res. 32 (2002) 409.

[20] A. Damasceni, L. Dei, E. Fratini, F. Ridi, S.-H. Chen, P. Baglioni, J. Phys. Chem. B 106 (2002) 11572.

[21] F. Ridi, P. Luciani, E. Fratini, P. Baglioni, J. Phys. Chem. B 113 (2009) 3080.

[22] F. Ridi, E. Fratini, S. Milani, P. Baglioni, J. Phys. Chem. B 110 (2006) 16326.

[23] V. Bortolotti, P. Fantazzini, R. Mongiorgi, S. Sauro, S. Zanna, Cem. Concr. Res. 42 (2012) 577.

[24] E. Leutheusser, Phys. Rev. A 29 (1984) 2765.

[25] W. Götze, L. Sjögren, Rep. Prog. Phys. 55 (1992) 241.

[26] S.-H. Chen, C. Liao, F. Sciortino, P. Gallo, P. Tartaglia, Phys. Rev. E 59 (1999) 6708.

[27] L. Liu, A. Faraone, C.-Y. Mou, C.-W. Yen, S.-H. Chen, J. Phys. Condens. Matter 16 (2004) S5403. 
[28] L. Liu, S.-H. Chen, A. Faraone, C.-W. Yen, C.-Y. Mou, Phys. Rev. Lett. 95 (2005) 117802.

[29] L. Liu, S.-H. Chen, A. Faraone, C.-W. Yen, C.-Y. Mou, A.I. Kolesnikov, E. Mamontov, J. Leão, J. Phys. Condens. Matter 18 (2006) S2261.

[30] A. Faraone, L. Liu, C.-Y. Mou, C.-W. Yen, S.-H. Chen, J. Chem. Phys. 121 (2004) 10843.

[31] Z. Wang, E. Fratini, M. Li, P. Le, E. Mamontov, P. Baglioni, S.-H. Chen, Phys. Rev. E 90 (2014) 042705.

[32] Z. Wang, P. Le, K. Ito, J.B. Leão, M. Tyagi, S.-H. Chen, J. Chem. Phys. 143 (2015) 114508.

[33] E. Mamontov, K.W. Herwig, Rev. Sci. Instrum. 82 (2011) 085109.

[34] F. Ridi, E. Fratini, P. Baglioni, J. Colloid Interface Sci. 357 (2011) 255.

[35] H.M. Jennings, Cem. Concr. Res. 30 (2000) 101.

[36] H.M. Jennings, Cem. Concr. Res. 38 (2008) 275.

[37] W.-S. Chiang, E. Fratini, P. Baglioni, D. Liu, S.-H. Chen, J. Phys. Chem. C 116 (2012) 5055.

[38] W.-S. Chiang, E. Fratini, F. Ridi, S.-H. Lim, Y.-Q. Yeh, P. Baglioni, S.M. Choi, U.S. Jeng, S.-H. Chen, J. Colloid Interface Sci. 398 (2013) 67.

[39] E. Mamontov, H. O’Neill, Q. Zhang, W. Wang, D.J. Wesolowski, J. Phys. Condens. Matter 24 (2012) 064104.

[40] A.J. Allen, J.J. Thomas, Cem. Concr. Res. 37 (2007) 319.

[41] J.J. Thomas, H.M. Jennings, A. J. Allen, Cem. Concr. Res. 28 (1998) 897.

[42] J.J. Thomas, J.J. Chen, A.J. Allen, H.M. Jennings, Cem. Concr. Res. 34 (2004) 2297.

[43] S.-H. Chen, Y. Zhang, M. Lagi, S.-H. Chong, P. Baglioni, F. Mallamace, J. Phys. Condens. Matter 21 (2009) 504102.

[44] C.E. Bertrand, K.-H. Liu, E. Mamontov, S.-H. Chen, Phys. Rev. E 87 (2013) 042312.

[45] M. Fourmentin, P. Faure, S. Gauffinet, U. Peter, D. Lesueur, D. Daviller, G. Ovarlez, P. Coussot, Cem. Concr. Res. 77 (2015) 1-8.

[46] N.M. Nemes, D. A. Neumann, R. A. Livingston, J. Mater. Res. 21 (2006) 2516-2523.

[47] V.K. Peterson, D. A. Neumann, R. A. Livingston, Phys. B Condens. Matter 385-386 (2006) 481-486. 


\section{Graphical Abstract}

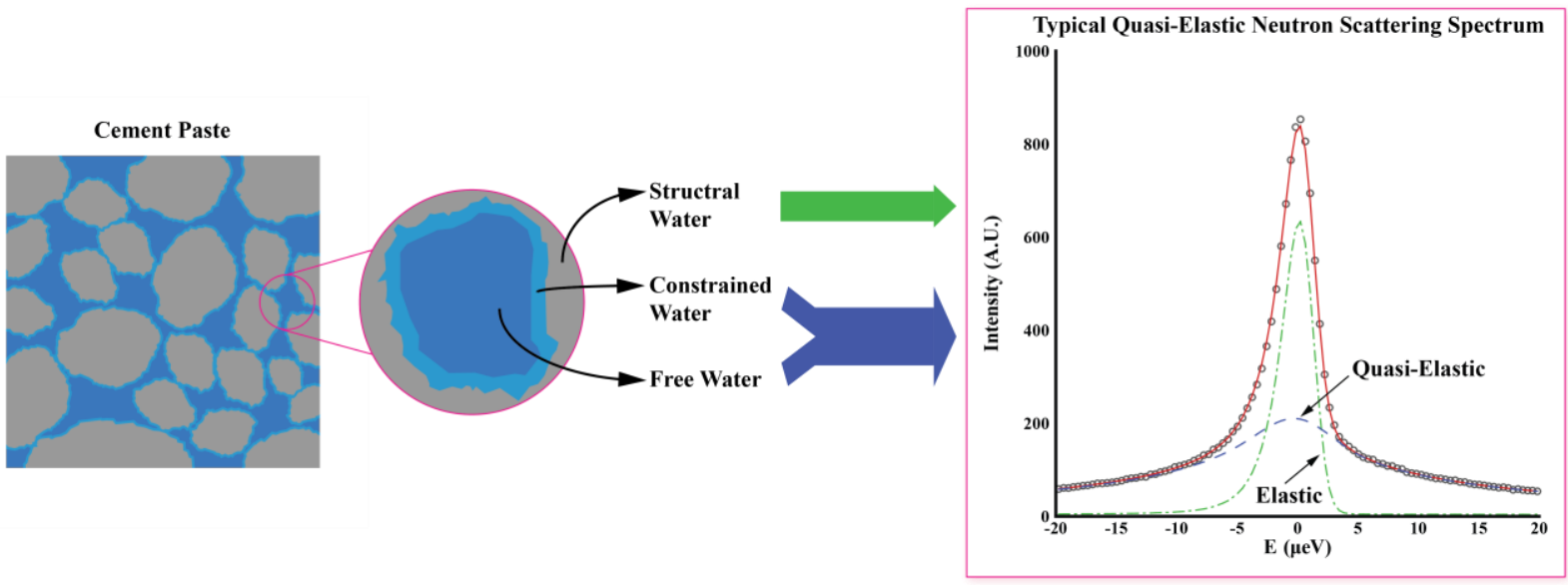

